\title{
Aplicação pós-colheita de luz ultravioleta (uv-c) em pêssegos cultivar Jade, armazenados em condição ambiente
}

\author{
UV-C light on the preservation of peaches cv. Jade at room temperature \\ Enilton Fick Coutinho ${ }^{1}$ Joel Lima da Silva Junior ${ }^{2}$ Janni André Haerter ${ }^{3}$ \\ Gilson Ribeiro Nachtigall ${ }^{3}$ Rufino Fernando Flores Cantillano ${ }^{1}$
}

\section{RESUMO}

Avaliaram-se os efeitos do fungicida benomil $(0,06 \%)$ e da luz ultravioleta $(254 \mathrm{~nm}, U V-C)$ na conservação de pêssegos cultivar Jade, aos 4 e 8 dias de armazenamento em condição ambiente $\left(26^{\circ} \mathrm{C}\right.$ e $75-85 \%$ de UR). Os tratamentos foram: $T_{1}$-Testemunha; $T_{2}$ - benomil $(0,06 \%)$; $T_{3}-10$ minutos de exposição à radiaçãa $U V-C \quad\left(3,71 \mathrm{kj.} \mathrm{m}^{-2}\right)$ $e^{3} T_{4}-30$ minutos de exposição à radiação $U V-C \quad\left(11,15 \mathrm{kj.} \mathrm{m}^{-2}\right)$. $O$ delineamento experimental foi o inteiramente casualizado, seguindo-se um esquema fatorial $2 \times 4$ com 3 repetições de 10 frutos cada. Os parâmetros avaliados foram: sólidos solúveis totais (SST), acidez titulável total (ATT), $p H$, firmeza da polpa e incidência de podridão. Os pêssegos não apresentaram alteração do $\mathrm{pH}$, em ambos os tempos de avaliação. OS SST, a ATT e a firmeza da polpa sofreram alterações aos 4 dias de armazenamento, sendo que, aos 8 dias nessa condição, a firmeza da polpa e a incidência de podridão apresentaram diferenças significativas em função dos tratamentos. Os pêssegos dos tratamentos $T$ e $T$ apresentaram menor incidência de podridões aos 8 días dé armazenamento, porém não diferiram estatisticamente entre si. Concluiu-se que as alterações nos parâmetros físicoquímicos (SST, ATT, pH e firmeza da polpa), aos 4 e 8 dias de armazenamento ambiente $\left(26^{\circ} \mathrm{C}\right.$ e $75-80 \%$ de UR), não afetaram a qualidade dos pêssegos bem como a utilização de luz ultravioleta $(254 \mathrm{~nm}, U V-C)$, durante 30 minutos, controla $100 \%$ as podridões dos pêssegos da cultivar Jade, armazenados, após 4 e 8 dias, em condição ambiente.

Palavras-chave: Prunus persica, benomil, luz ultravioleta$C$, armazenamento em condição ambiente.

\section{ABSTRACT}

Treatments with benomyl and UV light were evaluated with regard to the ocurrence of fruit rot of peaches $c v$. Jade kept under room temperature conditions for 8 days after harvesting. The evaluations were performed at the $4^{\text {th }}$ and $8^{\text {th }}$ days at room conditons $\left(26^{\circ} \mathrm{C}\right.$ and $\left.75-80 \% \quad R H\right)$. The treatments were: $T_{1}$-control; $T_{2}$-peaches immersed in $0,06 \%$ benomyl water solution; $T_{3}$-peaches exposed to $U V$-C light $\left(3,71 \mathrm{kj} \cdot \mathrm{m}^{-2}\right)$ for 10 minutes ; and $T_{4}$ - peaches exposed for 30 minutes to the UV-C light $\left(11,15 \mathrm{kj}^{4} \mathrm{~m}^{2}\right)$. The experiment a $2 \times 4$ factorial arrangementa in design was a complete randomized with three replications of ten peaches per plot. The variables evaluated were: total soluble solids (TSS); total titrable acidity (TTA); $p H$; firmness and incidence of rots. The $\mathrm{pH}$ of the peaches did not change during 4 or 8 days they remained at room temperature. The level of TSS and TTA was changed at $4^{\text {th }}$ day, but only the firmness of the fruit had significant differences between treatments at the $8^{\text {th }}$ day at room conditions. The peaches of $T$ and $T$ treatments had the least incidence of rots. It was concluded that the changes in TSS, TTA, pH and firmness, after 4 and 8 root condition $\left(26^{\circ} \mathrm{C}\right.$ and $75-80 \%$ RH) due to treatments did not affect the final quality of peaches. The UV-C, 254nm light treatment for thirty minutes controls rot incidence up to $100 \%$ after 4 and 8 days in preservation at root condition.

Key words: Prunus persica, UV light, benomyl, room condition.

\section{INTRODUÇÃO}

O pêssego (Prunus persica (L.) Batsch.) é um fruto que apresenta pequeno período de armazenamento, devido a problemas tais como: rápida desidratação, elevada perda da firmeza da polpa e incidência de fungos causadores de podridões na póscolheita.

Para evitar o aparecimento de podridões no armazenamento dos frutos, tem sido recomendada a

${ }^{1}$ Engenheiro Agrônomo, Doutor, Pesquisador da Embrapa Clima Temperado. E-mail: enilton@cpact.embrapa.br. Autor para correspondência.

${ }^{2}$ Médico Veterinário, MSc., Universidade Nilton Lins, Manaus-AM.

${ }^{3}$ Engenheiro Agrônomo, MSc., Doutorando em Fruticultura de Clima Temperado, Universidade Federal de Pelotas. 
utilização de fungicidas, principalmente benomil e tiabendazole. No entanto, estes produtos podem ser deletérios à saúde do homem e ao meio ambiente.

Vários pesquisadores têm procurado alternativas para controlar doenças que incidam na pós-colheita de frutos.

STEVENS et al. (1996) utilizaram baixas doses de luz ultravioleta (254 nm, UV-C) para controlar podridões na pós-colheita de pêssegos, maçãs, pomelos e tangerinas. Eles verificavam que as mesmas foram eficientes para o controle da Monilinia fructicola em pêssegos; Alternaria spp, Colletotrichum gloeosporoides e Monilinia spp em maçãs; Penicillium digitatum em pomelos e tangerinas; Alternaria citri e Geotrichum candidum em tangerinas.

D'HALLEWIN et al. (1994) verificaram que a luz ultravioleta (254 nm, UV-C) foi eficiente para controlar podridões pós-colheita de tangerinas "Avana".

A luz ultravioleta tem sido utilizada como alternativa no controle de podridões pós-colheita em pêssegos (CRISOSTO et al., 1998), maçãs, pomelos, tangerinas (STEVENS et al., 1996) e tomates (LIU et al., 1993).

No Brasil, não são comuns, na literatura, trabalhos sobre a utilização da luz ultravioleta no controle de doenças incidentes na pós-colheita de pêssegos destinados ao consumo in natura ou para industrialização.

O objetivo deste trabalho foi verificar a influência e a eficiência da luz ultravioleta (UV-C) e do fungicida benomil no controle de podridões e na qualidade de pêssegos da cv. Jade, armazenados em condição ambiente $\left(26^{\circ} \mathrm{C}\right.$ e $75-80 \%$ de UR) durante $4 \mathrm{e}$ 8 dias.

\section{MATERIAL E MÉTODOS}

O trabalho foi realizado na Embrapa Clima Temperado, Pelotas/RS. Utilizaram-se pêssegos da cultivar Jade, colhidos em 13/12/1999, no estádio de maturação de vez ( $25 \%$ de coloração amarela) e com a seguinte caracterização físico química: sólidos solúveis totais (SST $11,5^{\circ}$ Brix), acidez titulável total (ATT) em ácido málico (1,68\%), pH $(3,7)$, firmeza da polpa (6,9 libras).

Os frutos foram selecionados pelo tamanho (diâmetro equatorial), sendo classificados como Tipo I $(\geq 57 \mathrm{~mm})$ e, em seguida, foram submetidos aos seguintes tratamentos: $\mathrm{T}_{1}$-Testemunha ; $\mathrm{T}_{2}$-benomil $(0,06 \%) ; \mathrm{T}_{3}-10$ minutos de exposição à radiação UV-C $\left(3,71 \mathrm{kj} . \mathrm{m}^{2}\right)$ e $\mathrm{T}_{4}-30$ minutos de exposição à radiação
UV-C $\left(11,15 \mathrm{kj} \mathrm{m}^{2}\right)$. O tratamento com luz ultravioleta foi realizado com lâmpada G15T8 de $15 \mathrm{~W}$, comprimento de onda de $254 \mathrm{~nm}$, instalada em capela de madeira revestida com papel alumínio. Os frutos foram, imaginariamente, divididos em duas faces e cada face ficou posicionada com a cavidade peduncular distanciada $20 \mathrm{~cm}$ da fonte de luz, sendo expostos por igual tempo à radiação ultravioleta. Após receberem os tratamentos, os frutos foram embalados em bandejas de polietileno expandido (4 frutos/bandeja), envolvidos em filme de polietileno e armazenados em ambiente com temperatura entre $23^{\circ} \mathrm{C}$ e $26^{\circ} \mathrm{C}$, umidade relativa entre $72 \%$ e $85 \%$ monitoradas por termohigrógrafo THG 1.

Avaliaram-se os seguintes parâmetros: a) Teor de SST (expresso em ${ }^{\circ}$ Brix); determinado por meio de refratometria, com correção da temperatura para $20^{\circ}$ C; b) ATT (expressa em \% de ácido málico), determinada por titulometria com $\mathrm{NaOH}$ a $0,1 \mathrm{~N}$ até $\mathrm{pH}$ 8,1 ; c) $\mathrm{pH}$ da polpa triturada, determinado com peagâmetro (Digimed DMPH 2); d) Firmeza da polpa, determinada com penetrômetro manual Mc Cornick, modelo FT 327, com ponteira de 5/16" de diâmetro, fazendo-se duas leituras em lados opostos, após a remoção da casca; e) Incidência de podridões, avaliada, visualmente, pelos sintomas na epiderme, utilizandose escala de notas desenvolvida pelos autores, sendo 1 (sem podridões), 2 (25\%), 3 (50\%), 4 (75\%) e 5 $(100 \%)$ da superfície do fruto com incidência de podridões).

O delineamento experimental adotado foi o inteiramente casualizado, seguindo-se um esquema fatorial 2 x 4, com 3 repetições de 10 frutos cada. Estudou-se o efeito do fator tratamentos, sendo realizada a avaliação dos parâmetros estudados aos 4 e 8 dias de armazenamento.

Para verificar o efeito dos tratamentos nos parâmetros avaliados, utilizou-se análise da variância e teste de comparação múltipla de médias (Duncan a $5 \%$ de probabilidade). Os dados percentuais originais foram transformados em arco seno da raiz quadrada de $\mathrm{x} / 100$.

\section{RESULTADOS E DISCUSSÃO}

\section{Análises físico-químicas dos frutos}

O teor de SST dos pêssegos, aos 4 dias de armazenamento, foi maior no tratamento $4\left(12,6^{\circ}\right.$ Brix), diferenciando-se significativamente da testemunha (11, $9^{\circ}$ Brix) e do tratamento com benomil (11,8 $8^{\circ}$ Brix). Aos 8 dias de armazenamento, não houve diferença significativa entre os tratamentos. A ATT dos frutos, aos 4 dias de armazenamento, foi maior 
na testemunha $(1,497 \%)$, diferindo significativamente apenas do tratamento $4(1,268 \%)$. Os tratamentos não apresentaram diferença significativa aos 8 dias de armazenamento. $\mathrm{O}$ pH dos pêssegos não apresentou variação significativa entre os tratamentos, aos 4 e 8 dias de armazenamento, tendo, porém, como valor máximo 3,49 para o tratamento 4 , aos 4 dias de armazenamento, e 3,69 para o tratamento 3, aos 8 dias de armazenamento. Com relação à firmeza da polpa, os pêssegos tratados com benomil apresentaram-se mais firmes em ambos os tempos de armazenamento ( 4 dias $=7,16$ libras e 8 dias $=6,60$ libras) $($ Tabela 1$)$.

Os resultados da análise físico-química são corroborados por aqueles obtidos por HAERTER (1995), que verificou que pêssegos das cultivares Jade e Diamante perdem rapidamente a qualidade, devido ao quase completo amadurecimento, principalmente aqueles colhidos no estádio de maturação verdoengo (“de vez”).

Segundo TAVARES et al. (1991), a modificação da atmosfera de armazenamento, mediante o envolvimento dos frutos com sacos plásticos reduz as perdas de peso, porém não evita que ocorram reduções na firmeza da polpa.
As variações dos valores de SST, ATT e firmeza da polpa nos diferentes tratamentos, aos 4 e 8 dias de armazenamento, ocorreram, possivelmente, devido a utilização dos SST (constituídos por compostos solúveis em água, tais como açúcares, ácidos, vitamina c, aminoácidos e algumas pectinas) e ATT (constituída por ácidos orgânicos) no metabolismo respiratório. D’HALLEWIN et al. (1994) observaram variações da ATT, dos SST e do pH em tangerinas cultivar Avana, quando tratadas com luz ultravioleta (254nm, UV-C), água quente e tiabendazole (TBZ), e armazenadas a $2^{\circ} \mathrm{C}$ e $8^{\circ} \mathrm{C}$, durante um mês.

\section{Incidência de podridões}

Aos 4 dias de armazenamento, os pêssegos não apresentaram sintomas de ataque de podridões (nota 1), entretanto, aos 8 dias, aqueles dos tratamentos $\mathrm{T}_{4}$ e $\mathrm{T}_{2}$ apresentaram-nos em maior quantidade, nota 1 e 1,25, respectivamente, embora, não diferissem estatisticamente entre si (Tabela1).

STEVENS et al. (1996) verificaram que pêssegos da cultivar Elberta apresentaram menor incidência de Monilinia fructicola (1\%), aos 10 dias de armazenamento a $12^{\circ} \mathrm{C}$, quando tratados com luz ultravioleta $(254 \mathrm{~nm}, \mathrm{UV}-\mathrm{C})$ nas dosagens de 4,8 e 7,

Tabela 1 - Características físicas e químicas e incidência de podridões, em função do tempo de armazenamento e dos diferentes tratamentos, em pêssegos (Prunus persica (L.) Batsch) cv. Jade, armazenados em condição ambiente $\left(26^{\circ} \mathrm{C}\right.$ e $75-80 \%$ de UR). Embrapa Clima Temperado, Pelotas-RS.

\begin{tabular}{|c|c|c|c|c|c|c|c|c|c|c|}
\hline \multirow{3}{*}{$\begin{array}{l}\text { Parâmetros avaliados } \\
\left.\text { SST ( }{ }^{\circ} \text { Brix }\right)\end{array}$} & \multirow{3}{*}{$\begin{array}{l}\text { Tratamentos } \\
\text { Testemunha }\end{array}$} & \multicolumn{7}{|c|}{ Tempo de armazenamento (dias) ${ }^{1}$} & \multirow{3}{*}{$\begin{array}{c}\text { Média geral } \\
11,79\end{array}$} & \multirow{3}{*}{$\begin{array}{l}\text { CV (\%) } \\
2,7288\end{array}$} \\
\hline & & \multicolumn{3}{|c|}{4} & \multicolumn{4}{|c|}{8} & & \\
\hline & & 11,96 & & $\mathrm{~b}$ & 11,65 & $\mathrm{a}$ & & & & \\
\hline & benomil $(0,06 \%)$ & 11,83 & & $\mathrm{~b}$ & 11,25 & $\mathrm{a}$ & & & & \\
\hline & UV C $10 \mathrm{~min}$. & 12,36 & $\mathrm{a}$ & $\mathrm{b}$ & 11,55 & $\mathrm{a}$ & & & & \\
\hline & UV C $30 \mathrm{~min}$. & 12,63 & $\mathrm{a}$ & & 11,74 & $\mathrm{a}$ & & & & \\
\hline \multirow{4}{*}{$\begin{array}{l}\text { ATT }(\% \text { de } \\
\text { ácido málico })\end{array}$} & Testemunha & 1,497 & $\mathrm{a}$ & & 1,190 & $\mathrm{a}$ & & & 13,19 & 9,1902 \\
\hline & benomil $(0,06 \%)$ & 1,347 & $\mathrm{a}$ & $\mathrm{b}$ & 1,222 & $\mathrm{a}$ & & & & \\
\hline & UV C $10 \mathrm{~min}$. & 1,390 & $\mathrm{a}$ & $\mathrm{b}$ & 1,082 & a & & & & \\
\hline & UV C $30 \mathrm{~min}$. & 1,268 & & $\mathrm{~b}$ & 1,195 & $\mathrm{a}$ & & & & \\
\hline \multirow[t]{4}{*}{$\mathrm{pH}$} & Testemunha & 3,48 & $\mathrm{a}$ & & 3,67 & $\mathrm{a}$ & & & 3,59 & 1,3275 \\
\hline & benomil $(0,06 \%)$ & 3,45 & $\mathrm{a}$ & & 3,68 & $\mathrm{a}$ & & & & \\
\hline & UV C $10 \mathrm{~min}$. & 3,48 & $\mathrm{a}$ & & 3,69 & $\mathrm{a}$ & & & & \\
\hline & UV C $30 \mathrm{~min}$. & 3,49 & $\mathrm{a}$ & & 3,62 & $\mathrm{a}$ & & & & \\
\hline \multirow{4}{*}{$\begin{array}{l}\text { Firmeza da } \\
\text { polpa (libras) }\end{array}$} & Testemunha & 6,15 & $\mathrm{a}$ & & 4,90 & & $\mathrm{~b}$ & $\mathrm{c}$ & 5,83 & 7,4045 \\
\hline & benomil $(0,06 \%)$ & 7,16 & $\mathrm{a}$ & & 6,30 & $\mathrm{a}$ & & & & \\
\hline & UV C $10 \mathrm{~min}$. & 5,82 & & $\mathrm{~b}$ & 5,37 & & $\mathrm{~b}$ & & & \\
\hline & UV C 30 min. & 5,45 & & $\mathrm{~b}$ & 4,40 & & & $\mathrm{c}$ & & \\
\hline Incidência de & Testemunha & 1,00 & $\mathrm{a}$ & & 1,75 & $\mathrm{a}$ & & & 1,19 & 18,2321 \\
\hline \multirow[t]{3}{*}{ Podridão } & benomil $(0,06 \%)$ & 1,00 & $\mathrm{a}$ & & 1,25 & & b & $\mathrm{c}$ & & \\
\hline & UV C $10 \mathrm{~min}$. & 1,00 & $\mathrm{a}$ & & 1,50 & $\mathrm{a}$ & $\mathrm{b}$ & & & \\
\hline & UV C $30 \mathrm{~min}$. & 1,00 & $\mathrm{a}$ & & 1,00 & & & $\mathrm{c}$ & & \\
\hline
\end{tabular}

1 = Médias não seguidas da mesma letra na coluna, diferem entre si pelo teste de Duncan em 5\% de probabilidade de erro.

Ciência Rural, v. 33, n. 4, jul-ago, 2003. 
$50 \mathrm{kj} \mathrm{m}^{-2}$, na safra 1989. Porém, no ano seguinte, com a mesma cultivar, as menores incidências de Monilinia fructicola ocorreram em pêssegos tratados com UV$\mathrm{C}$ nas doses de $7,5 \mathrm{kj} \mathrm{m}^{-2}(7 \%)$ e $20 \mathrm{kj} \mathrm{m}^{-2}(5 \%)$.

D'HALLEWIN et al. (1994), ao utilizarem luz ultravioleta $(254 \mathrm{~nm}$, UV-C) para controlar podridões pós-colheita em tangerinas, cultivar Avana, armazenadas a $8^{\circ} \mathrm{C}$ e $2^{\circ} \mathrm{C}$, durante um mês, seguido de uma semana a $17^{\circ} \mathrm{C}$, observaram que a luz UV reduziu significativamente a incidência de podridões $(1,67 \%)$, quando comparada à testemunha $(3,49 \%)$.

A utilização de luz ultravioleta no controle de podridões pós-colheita de pêssegos, necessita de novos estudos. Os autores sugerem melhorias na metodologia, principalmente com relação à utilização de inoculação artificial dos frutos com Monilinia fructicola e também avaliações quanto à perda de peso, atividade respiratória $\left(\mathrm{CO}_{2}\right.$ e etileno $)$ e desordens fisiológicas que sejam realizadas em novos experimentos.

\section{CONCLUSÕES}

As alterações nos parâmetros físicoquímicos (STT, ATT, pH e firmeza da polpa), aos 4 e 8 dias de armazenamento ambiente $\left(26^{\circ} \mathrm{C}\right.$ e $75-80 \%$ de UR), não afetam a qualidade de pêssegos.

A utilização de luz ultravioleta $(254 \mathrm{~nm}$, UVC) durante 30 minutos, controla em $100 \%$ as podridões de pêssegos da cultivar Jade, aos 4 e 8 dias de armazenamento.

\section{REFERÊNCIAS BIBLIOGRÁFICAS}

CRISOSTO, C. H. et al. Comparing pulsated ultraviolet light and postharvest fungicide for peach fruit decay control. Acta Horticulturae, Wellington, v.2, n.465, p.471-479, 1998.

D'HALLEWIN, G. et al. Reducing decay of Avana mandarin fruit by the use of UV, heat and thiabendazole treatments. Acta Horticulturae, Wellington, n. 368 , p.387-394, 1994.

HAERTER, J.A. Influência do ponto de colheita e da refrigeração no amadurecimento de pêssegos (Prunus persica (L.) Batsch), cvs. Diamante e Jade. 1995. 82f. Dissertação (Mestrado em Agronomia) - Curso de Fruticultura de Clima Temperado, Universidade Federal de Pelotas.

LIU, J. et al. The effect of ultraviolet C light on storage rots and ripening of tomatoes. Journal Food Protection, Great Britain, n.56, p.868-972, 1993.

PASQUALI, P. Efeito do uso de cera e filmes de polietileno durante a frigoconservação de pêssegos (Prunus persica (L.) Batsch). 1993. $107 \mathrm{f}$. Dissertação (Mestrado em Agronomia) - Curso de Fruticultura de Clima Temperado, Universidade Federal de Pelotas.

STEVENS, C. et al. Plant hormesis induced by ultraviolet light $\mathrm{C}$ for controlling postharvest diseases of tree fruits. Crop Protection, Ames IA, v.15, n.2, p.129-134, 1996.

TAVARES, L.B.B. ; CHITARRA, M.I.F.; CHITARRA, A.B. Using modified in the storage of two peaches cvs. (Prunus persica (L.) Batsch). Potential of conservation and quality. Arquivos de Biologia e Tecnologia, Curitiba, v.34, n.3/4, p.401-413,1991. 